\title{
Análise do conforto térmico nos projetos de enfermarias hospitalares nos trópicos: uma revisão sistemática
}

Introdução: A qualidade do projeto arquitetônico depende de uma variedade de fatores que lhes dão forma e significado. $\mathrm{O}$ ambiente construído, entre eles 0 hospitalar, necessita de padrões estéticos, ergonômicos, acústicos, visuais, higrotérmicos, psicológicos e da qualidade do ar. Ademais, deve-se evidenciar o impacto na qualidade do atendimento pelo bem-estar do profissional, bem como dos pacientes internados e ambulatoriais. Objetivo: Averiguar as diferenças obtidas nas pesquisas em países tropicais com os parâmetros esperados pelo ASHRAE e ISO 7730. Metodologia: Trata-se de um estudo multicêntrico realizado entre a Universidade Federal da Paraíba (UFPB) e o Centro Universitário de João Pessoa (UNIPE), a partir de uma abordagem interdisciplinar e multiprofissional entre a medicina e a arquitetura através de uma revisão sistemática. Tem um caráter qualitativo, descritivo, e foi realizado entre janeiro de 2019 e janeiro de 2020 , através das bases de dados PubMED/MEDLINE, Crossref, Scopus, CAPES, Web of Science e Science Direct, incluindo um total de dez trabalhos. Resultados e Discussão: Percebe-se que há uma diferença significativa em todas as pesquisas que buscaram diferenciar a temperatura neutra utilizando-se o TSV e o PMV. Sendo a variação maior do que $1^{\circ} \mathrm{C}$ e chegando até $3,1^{\circ} \mathrm{C}$ de diferença. Ademais, os padrões de conforto térmico estabelecidos pelo padrão PMV da ASHRAE-55 não representam ambientes hospitalares em países com clima tropical ou subtropical. Conclusão: Compreende-se que devem ser realizadas pesquisas locais visando adequar os padrões de temperatura às populações que nela habitam.

Palavras-chave: Conforto ambiental; Hospital; Ambiente construído.

\section{Analysis of thermal comfort in hospital nursing projects in the tropics: a systematic review}

\begin{abstract}
Introduction: The quality of architectural design depends on a variey of factors that give it shape and meaning. The built environment, incluindo the hospital, requires aesthetic, ergonomic, acoustic, visual, termal, psychological and air quality standards. In addition, the impacto $n$ the quality of care for the well-being of the professional, as well as impatients and outpatients, must be evidenced.Objective: To investigate the diferences obtained in surveys in tropical countries with the parameters expected by ASHARE and ISSO 7730. Methodology: This work is a multicenter study carried out between the Federal University of Paraiba (UFPB) and the University Center of João Pessoa (UNIPE), based on na interdisciplinar and multiprofessional approach between medicine and architecture through a review systematic, qualitative, descriptive, carried out between january 2017 and january 2020, through the PubMED/MEDLINE, Crossref, Scopus, CAPES, Web of Science and Science Direct databases, including a total of ten works.. Results and Discussion: It is noticed that there is a significant difference in all studies that sought to differentiate the neutral temperature using TSV and PMV. The variation being greater than $1^{\circ} \mathrm{C}$ and reaching up to $3,1^{\circ} \mathrm{C}$ difference. Furthermore, the thermal comfort standards established by the ASHRAE-55 PMV standard do not represent hospital environments in countries with a tropical or subtropical climate Conclusion: Thus, it is understood that local research should be carried out in order to adapt the temperature standards to the populations that livre there.
\end{abstract}

Keywords: Environmental comfort; Hospital; Built environment.

Topic: Desenvolvimento, Sustentabilidade e Meio Ambiente

Reviewed anonymously in the process of blind peer

Maurus Marques de Almeida Holanda (iD Universidade Federal da Paraíba, Brasil http://lattes.cnpq.br/2786546058482445 http://orcid.org/0000-0003-2217-4434

maurusholandaa@hotmail.com

Kelly Christine Silva de Lima (iD)

Centro Universitário de João Pessoa, Brasil http://lattes.cnpq.br/5140017052432316 http://orcid.org/0000-0001-7611-7377

kelly.lima@unipe.edu.br

Daniel Meira Nóbrega de Lima

Universidade Federal da Paraíba, Brasil http://lattes.cnpq.br/8706125054242800

http://orcid.org/0000-0001-8384-3895

danielmrnobrega@gmail.com

d

DOI: 10.6008/CBPC2179-6858.2021.001.0046
Received: 01/01/2021

Approved: 28/01/2021

\author{
Lucas Almeida de Souza \\ Centro Universitário de João Pessoa, Brasil \\ http://lattes.cnpq.br/6437212846352386 \\ http://orcid.org/0000-0003-2863-696X \\ lucasalmeidasouz@gmail.com \\ Artur Roosevelt Cruz de Macêdo Feitosa \\ Universidade Federal da Paraíba, Brasil \\ http://lattes.cnpq.br/2229535898926349 \\ http://orcid.org/0000-0003-1366-2540 \\ arthur.arturr@gmail.com
}

Referencing this:

HOLANDA, M. M. A.; LIMA, K. C. S.; LIMA, D. M. N.; SOUZA, L. A.; FEITOSA, A. R. C. M.. Análise do conforto térmico nos projetos de enfermarias hospitalares nos trópicos: uma revisão sistemática. Revista Ibero Americana de Ciências Ambientais, v.12, n.1, p.570580, 2021. DOI: http://doi.org/10.6008/CBPC2179 6858.2021.001.0046 


\section{INTRODUÇÃO}

A multidimensionalidade do conceito de conforto remete a aspectos físicos, espirituais, sociais, psicológicos e ambientais (MUSSI, 1996). Assim, compreender holisticamente esse fenômeno construído a partir da transformação do espaço pela ação humana vem sendo fundamental para alcançarmos as necessidades da população (SANTOS, 2006; SANTOS, 2009; SANTOS, 2014).

Apesar de recentes avanços nas neurociências acerca da percepção multissensorial e afetiva do ambiente interno - que perpassa o conforto higrotérmico, acústico, visual, ambiental interno -, ainda não foram completamente elucidadas as minúcias das interações entre ambiente-homem (YANG et al., 2019).

Entendemos que o homem busca sempre a melhoria da sua qualidade de vida, modificando seus ambientes internos de forma que possa ter conforto. Além disso, a saúde humana está intrinsecamente correlacionada ao conforto ambiental ao qual o homem está inserido. Dessa forma, todo agente que afete o equilíbrio do ambiente tem impactos importantes na vida do usuário, devendo ser alterado o projeto ou consertada a falha técnica da edificação (HOOR et al., 2016).

Esses impactos negativos ainda não inteiramente conhecidos, mas muito já estudados e investigados nos mais diversos ambientes internos relacionados aos mais diversos confortos (HYAKYUNG et al., 2000; MENDELL et al., 2002; ROELOFSEN, 2002; VIOLA et al., 2008; MACNAUGHTON et al., 2017; ABBASI et al., 2018).

Sabe-se, também, da capacidade de interferência de uma área do conforto sobre outra, contudo, recentes estudos demonstram ainda lacunas importantes sobre esse conhecimento, sendo assim, necessárias novas pesquisas que possam averiguar uma melhor correlação entre os limites de cada conforto, de forma a encontrar para além das normas técnicas, a percepção do usuário e, assim, um nível de conforto ambiental "equilibrado" (OCHOA et al., 2002; FRONTCAZK et al., 2012). Considerando, outrossim, que o psicológico e a afetividade modificam a visão e percepção de conforto no ambiente que nos cerca (CASTALDO et al., 2018)

Além disso, faz-se necessário adequar a pesquisa às características da população que ela remete, uma vez que as populações de região temperada possuem idiossincrasias completamente distintas àquelas dos trópicos (CORBELLA, 2003; FERNANDES, 2006). Assim, há a necessidade de normas técnicas completamente diferentes, bem como a disparidade de percepções sobre o conforto do ambiente interno entre as regiões e entre a percepção e a norma (HAGHIGHAT, 1999; SATTAYAKORN et al., 2017). Dessa forma, percebeu-se em pós-estudos que há uma maior acurácia nas visões subjetivas do que as objetivas das normas técnicas para alcançar o conforto ambiental (GONZALEZ et al., 1997; FRANSSON et al., 2007).

Os hospitais são ambientes complexos onde transitam diversos tipos de pessoas (visitantes, pacientes, profissionais de saúde), cada um com suas respectivas vestimentas, gastos metabólicos, índices de massa corporal, além das variações individuais pelo sexo e idade. Assim, fornecer um conforto ambiental para cada um desses grupos se torna um desafio aos arquitetos, designers e engenheiros (OLESEN et al., 1984). 
Ademais, devemos salientar que essas edificações possuem características projetuais complexas, como uso de aparelho refrigerador ou de calefação e sistemas de ventilação e exaustão. Contudo, percebese que há poucos estudos relativos na área, sobretudo, quando se necessita de referenciais teóricos associados ao clima tropical, onde a amplitude térmica apesar de variável, apresenta temperaturas elevadas e valores expressivos de umidade relativa do ar em algumas regiões.

Entre os diversos confortos ambientais, temos o conforto térmico. Este tem impacto não apenas na sensação e prazer do indivíduo, mas também influencia diretamente na saúde e produtividade das pessoas no ambiente. E, na perspectiva hospitalar, este influencia desde o gasto metabólico do paciente, perda ou ganho de energia, e na multiplicação e inativação de microrganismos (WHO, 1990; KOSONEN et al., 2003).

No que tange o conforto térmico, é perceptível que os projetos arquitetônicos hospitalares na maioria dos países tropicais e subtropicais, têm seus padrões de qualidade muito abaixo aos esperados pelas normas nacionais e internacionais. Sendo as temperaturas objetivas e subjetivas muito distantes aos valores preconizados (HWANG et al., 2007; AZIZPOUR et al., 2012; WANG et al., 2012; AZIZPOUR et al., 2013; SATTAYAKOR et al., 2017)

Sabe-se também que a maioria dos parâmetros existentes, como o da American Society of Heating, Refrigerating and Air-Conditioning Engineers (ASHRAE), está em conformidade com padrões esperados aos países de climas temperados, onde há a maior quantidade de pesquisas na área, e condições ambientais equilibradas e opostas de acordo com as estações do ano. Dessa forma, diversas pesquisas em regiões tropicais e subtropicais (em especial com relação ao clima quente úmido) têm levantado a hipótese se há um nível de representatividade adequado a esses países (HWANG et al., 2007; YANG et al., 2008; HOOF, 2008). Vários pesquisadores têm demonstrado em suas respectivas regiões que as temperaturas, umidade, fluxo de ar, iluminação calculados pelas fórmulas e normas da ASHRAE e International Organization for Standardisation (ISO 7730/2005 - Ergonomics of the thermal environment) não condizem com as expectativas subjetivas esperadas pelas suas populações (HUMPHREYS, 1998; AZIZPOUR et al., 2013; SATTAYAKORN et al., 2017).

Para isso, têm sido levantadas várias estratégias que possam alcançar as expectativas esperadas nos projetos pelos pacientes, profissionais do local, e visitantes. Bem como, tem sido realizado uma análise energética, uma vez que o uso extensivo de aparelhos de refrigeração tem aumentado muito os gastos, no entanto há outras formas de atingirmos os padrões ideais.

Há, principalmente, dois parâmetros utilizados para avaliação de conforto térmico. O método mais antigo desenvolvido por Fanger (1970), através do Predicted Mean Vote (PMV) e do Predicted Percentage of Discomfort (PPD) (COUTINHO, 2005), que mais tarde tornaram-se a base para o ASHRAE 55. Este modelo por meio de determinação analítica, envolvendo parâmetros humanos (taxa de metabolismo da atividade e resistência do vestuário) e ambientais (temperatura do ar, temperatura média radiante, velocidade do ar e umidade relativa), fornece os padrões ambientais considerados aceitáveis para o conforto térmico geral, assim como o desconforto térmico geral. Atualmente, esses parâmetros aumentaram incluindo também sexo, idade, estado de saúde, adaptabilidade ao ambiente (HAVENITH, 2001; KARJALAINEN, 2007). 
O segundo método mais complexo, o Thermal Sensation Vote (TSV), baseia-se, além de tudo, em parâmetros subjetivos, psicológicos e culturais, através de votos a partir de sensações sobre o conforto do indivíduo, sendo, assim, parâmetros diretos (HUMPHREYS, 2005). Deste modo, as previsões são mais fidedignas à demanda dos usuários, uma vez que a primeira se utiliza apenas de parâmetros analíticos de previsão, isto é, medidas indiretas.

Assim, esta pesquisa tem como objetivo averiguar as diferenças obtidas nas pesquisas em países tropicais com os parâmetros esperados pelo ASHRAE e ISO 7730. Elucidar as principais estratégias utilizadas para diminuir a temperatura, minimizando os gastos energéticos. Especificar os requisitos esperados por visitantes, profissionais de saúde e pacientes.

\section{METODOLOGIA}

Este presente trabalho trata-se de uma revisão sistemática, de natureza qualitativa e descritiva por meio das bases de dados da PubMED/MEDLINE, Crossref, Scopus, CAPES, Web of Science e Science Direct. A busca das produções científicas foi realizada no período de janeiro de 2019 e janeiro de 2020.

A análise foi voltada para as enfermarias que possuem grupos de expostos particulares, com necessidades específicas e períodos hospitalares prolongados envolvendo manhã e noite e, porventura, estações climáticas distintas. Além disso, a pesquisa focou em resultados encontrados em regiões tropicais e subtropicais.

Os critérios de inclusão definidos para seleção das publicações foram: 1) As publicações serem produzidas em inglês, português ou espanhol, com resumos disponíveis nessas bases de dados, de 2005 em diante, encontrados através das palavras-chave 'thermal comfort', 'hospital environment' e 'tropics'; 2) o tema central do estudo envolver conforto ambiental no ambiente hospitalar; 3) possuírem financiamento próprio ou de alguma instituição pública; 4) abordar enfermaria clínica ou cirúrgica do adulto; 5) abordar estudos no clima tropical ou subtropical; e 6) publicações de revista com fator de impacto maior do que 1.

Foram excluídas as publicações que não seguiram uma metodologia adequada com presença de viés sistemático em sua pesquisa, bem como artigos com baixo nível de evidência. Além disso, os trabalhos que estudassem apenas enfermaria pediátrica, enfermaria obstétrica, o bloco cirúrgico ou as Unidades de Terapia Intensiva (UTIS) como tema central também foram excluídos.

As informações colhidas nas publicações foram compiladas em um banco de dados através do Statistical Package for the Social Sciences (SPSS), versão 26.0, posteriormente, disposto em tabelas.

\section{RESULTADOS E DISCUSSÃO}

Ao realizar a pesquisa sistemática por meio das palavras-chave 'thermal comfort', 'hospital environment' e 'tropics' foram encontrados, inicialmente, cerca de 1068 publicações, entre as quais foram excluídas 260 por serem duplicadas. Em seguida, 808 estudos tiveram seus resumos lidos por completo, os quais foram excluídos 742 através dos critérios de inclusão e exclusão (figura 1). 


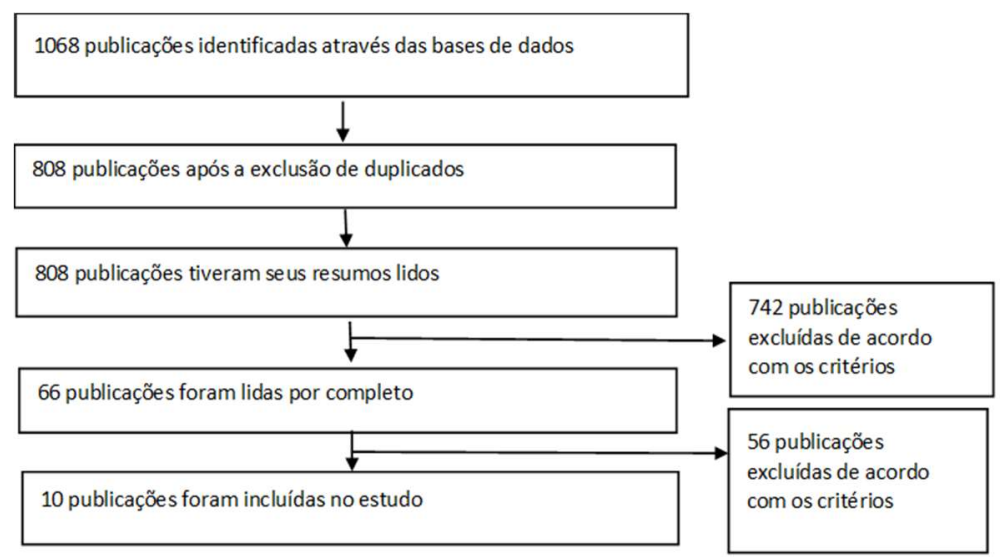

Figura 1: Fluxograma de estudos incluídos na revisão sistemática.

A partir disso, foi realizado a leitura completa de cada um dos sessenta e seis trabalhos, sendo excluídos cinquenta e seis. Dessa forma, foi incluído para o estudo final e discussão dos resultados, apenas dez publicações, as quais foram listadas na Tabela 1.

O padrão PMV da ASHRAE-55 possui algumas limitações em suas determinações analíticas, uma vez que o efeito isolante térmico dos materiais utilizados na norma, por inquéritos em edifícios residenciais e comerciais, é distinto aos encontrados em hospitais. Ademais, há uma dificuldade desse modelo em avaliar as modificações apresentadas pelos indivíduos em sua hospitalização, a partir da troca de vestimenta e diminuição da atividade metabólica. No entanto, a TSV permite que cada possibilidade seja calculada, de forma simples, direta e objetiva (ALOTAIBI et al., 2020).

Enquanto hospitais localizados em regiões com clima temperado possuem uma satisfação em faixas de temperatura mais próximas entre o paciente, o visitante e a staff (SKOOG et al., 2005). Em países tropicais, observou-se uma diferença significativa entre a faixa de temperatura satisfatória a equipe, em detrimento, àquela preferida pelos pacientes e visitantes, podendo alcançar, inclusive, uma insatisfação de $80 \%$ do segundo grupo, quando fixado os parâmetros segundo, apenas as necessidades da equipe de saúde (AZIZPOUR et al., 2012).

Dessa forma, percebeu-se que os padrões de conforto térmico estabelecidos pelo padrão PMV da ASHRAE-55 não representam ambientes hospitalares em países com clima tropical ou subtropical, sendo necessários mais estudos para representar melhor as necessidades dessa população (HWANG et al., 2007; AZIZPOUR et al., 2012; WANG et al., 2012 AZIZPOUR et al., 2013; NEMATCHUOA, 2017; SATTAYAKORN et al., 2017; ALOTAIBI et al., 2020). Na quase totalidade dos casos, observou-se uma insatisfação dos padrões de temperatura demonstrados por esses modelos analíticos (PMV), alcançando, geralmente, entre 30 a $50 \%$ de reprovação.

Os hospitais estudados estavam em desacordo tanto as normas nacionais quanto as esperadas pelos padrões da ASHRAE. Evidencia-se em alguns hospitais a conformidade de apenas 38 a $44 \%$ dos ambientes. Além disso, em alguns hospitais foi observado níveis de insatisfação com as condições térmicas, superiores as baseadas pelo PPD (POURSHAGHAGHY et al., 2012). Esses altos índices de insatisfação podem ser explicados também, em parte, devido à baixa estrutura física dos hospitais localizados nos trópicos, uma vez que essas regiões são compostas de países subdesenvolvidos ou em desenvolvimento, na maior parte. 
Diferentemente, dos hospitais bem equipados, que possuem controle térmico em cada unidade-quarto, hospitais sem infraestrutura, apenas, controlam a temperatura por sistema central (YAU et al., 2009).

Tabela 1: Publicações incluídas na revisão sistemática.

\begin{tabular}{|c|c|c|c|}
\hline Estudo e ano & $\begin{array}{l}\text { Base de } \\
\text { Dados }\end{array}$ & $\begin{array}{l}\text { Tipo de Estudo/País/ } n^{\circ} \text { de } \\
\text { participantes }\end{array}$ & Resultados \\
\hline $\begin{array}{l}\text { ALOTAIBI et al., } \\
2020\end{array}$ & $\begin{array}{l}\text { Science } \\
\text { Direct }\end{array}$ & $\begin{array}{l}\text { Transversal/Arábia } \\
\text { Saudita/120 participantes }\end{array}$ & $\begin{array}{l}\text { Há variações por sexo e tipo de enfermaria cirúrgica ou } \\
\text { clínica. Temperatura neutra é de } 25,6^{\circ} \mathrm{C} \text {, erro padrão de } \\
0,45^{\circ} \mathrm{C} \text {. Usando o método de Griffith houve uma faixa } \\
\text { aceitável muito grande } 16,2^{\circ} \mathrm{C} \text { a } 28,8^{\circ} \mathrm{C} \text {. Temperatura } \\
\text { neutra para homens }\left(25,3^{\circ} \mathrm{C}\right) \text {, mulheres }\left(26,6^{\circ} \mathrm{C}\right) \text {. } \\
\text { enfermaria cirúrgica }\left(24,8^{\circ} \mathrm{C}\right) \text { e enfermaria clínica } \\
\left(25,6^{\circ} \mathrm{C}\right)\end{array}$ \\
\hline
\end{tabular}

\begin{tabular}{llllll}
\hline $\begin{array}{l}\text { AZIZPOUR } \\
\text { 2012 }\end{array}$ & et & al., & Scopus & $\begin{array}{l}\text { Transversal/Malásia/110 } \\
\text { participantes }\end{array}$ & $\begin{array}{l}\text { Houve uma variação de } 1,8^{\circ} \mathrm{C} \text { entre a temperatura neutra } \\
\text { pelo TSV }(26,8) \text { e PMV }(25)\end{array}$ \\
\hline AZIZPOUR & et & al., & Crossref & $\begin{array}{l}\text { Transversal/Malásia/188 } \\
\text { participantes }\end{array}$ & $\begin{array}{l}\text { Há diferença de temperatura por com menos insatisfação e } \\
\text { preferência, sendo } 23,4^{\circ} \mathrm{C} \text { e } 20,3^{\circ} \mathrm{C}, \text { respectivamente. O }\end{array}$ \\
& & & & & $\begin{array}{l}\text { novo ponto neutro na escala de ASHARE passa }+0,7 \text { na } \\
\text { escala de } 7 \text { pontos. }\end{array}$
\end{tabular}

HWANG et al., 2007 Science $\quad$ Transversal/Taiwan/928 A falta de conforto térmico afeta respostas regulatórias. Há

Direct participantes

\begin{tabular}{|c|c|c|c|}
\hline KHALID et al., 2019 & CAPES & $\begin{array}{l}\text { Transversal/Malásia/389 } \\
\text { participantes }\end{array}$ & $\begin{array}{l}\text { Para os pacientes foi observado temperatura neutra efetiva } \\
\text { ideal de } 25,3^{\circ} \mathrm{C} \text { e para os visitantes } 25,5^{\circ} \mathrm{C}\end{array}$ \\
\hline $\begin{array}{l}\text { KHODAKARAMI e } \\
\text { KNIGHT, } 2008\end{array}$ & $\begin{array}{l}\text { Web of } \\
\text { Science }\end{array}$ & Série de casos/ Irã & $\begin{array}{l}\text { A partir da temperatura efetiva ideal } 24 \text { a } 26^{\circ} \mathrm{C} \text { poderia ser } \\
\text { reduzido as demandas de refrigeração e aquecimento }\end{array}$ \\
\hline $\begin{array}{l}\text { NEMATCHOUA } e t \\
\text { al., } 2017\end{array}$ & Scopus & $\begin{array}{l}\text { Transversal/ Madagascar/ } \\
298 \text { participantes (apenas } \\
\text { hospital) }\end{array}$ & $\begin{array}{l}\text { Os altos fluxos de ar natural. Foi observado que a } \\
\text { temperatura de conforto térmica ideal seria entre } 23,4^{\circ} \mathrm{C} \text { e } \\
26,8^{\circ} \mathrm{C} \text {, sendo a temperatura neutra efetiva de } 25,46^{\circ} \mathrm{C} \text {. } \\
\text { Valores muito próximos aos de ASHARE }\end{array}$ \\
\hline $\begin{array}{l}\text { SATTAYAKORN et } \\
\text { al., } 2017\end{array}$ & Crossref & $\begin{array}{l}\text { Transversal/Tailândia/928 } \\
\text { participantes }\end{array}$ & $\begin{array}{l}\text { Temperatura neutra observada para pacientes, visitantes e } \\
\text { funcionários foi quase a mesma, } 25,2^{\circ} \mathrm{C}, 25,1^{\circ} \mathrm{C} \text { e } 25,5^{\circ} \mathrm{C} \text {, } \\
\text { respectivamente. Participantes preferiam temperatura mais } \\
\text { fria a aquela neutra. }\end{array}$ \\
\hline WANG et al.,2012 & Scopus & $\begin{array}{l}\text { Transversal/Malásia/408 } \\
\text { participantes }\end{array}$ & $\begin{array}{l}\text { Faixa de temperatura operacional foi de } 22,9^{\circ} \mathrm{C} \text { a } 26,3^{\circ} \mathrm{C} \text {. } \\
\text { Sendo, } 1^{\circ} \mathrm{C} \text { abaixo da zona confortável prevista pela } \\
\text { ASHRAE }\end{array}$ \\
\hline YAU et al., 2009 & Pubmed & $\begin{array}{l}\text { Transversal/Malásia/ } \\
\text { participantes }\end{array}$ & $\begin{array}{l}\text { Temperatura neutra ideal para malaios era } 26,4^{\circ} \mathrm{C} \text {. E a } \\
\text { faixa ideal estava entre } 25,3^{\circ} \mathrm{C} \text { e } 28,2^{\circ} \mathrm{C} \text {. Temperaturas } \\
\text { mais altas são necessárias do que as especificadas pela } \\
\text { ASHRAE Diferença de temperatura ideal para pessoas } \\
\text { frágeis é } 0,3^{\circ} \mathrm{C} \text { mais alto do que as normais. Pessoas com } \\
\text { doenças crônicas são mais susceptíveis ao calor. }\end{array}$ \\
\hline
\end{tabular}

Percebe-se que há uma diferença significativa em todas as pesquisas que buscaram diferenciar a temperatura neutra utilizando-se o TSV e o PMV. Sendo a variação maior do que $0,95^{\circ} \mathrm{C}$ e chegando até $3,1^{\circ} \mathrm{C}$ de diferença (YAU et al., 2009; WANG et al., 2012; AZIZPOUR et al., 2012; AZIZPOUR et al., 2013; SATTAYARO 
et al., 2017)

Verificou-se através do modelo baseado no TSV, que a temperatura neutra para pacientes variou de 22,7 a $26,41^{\circ} \mathrm{C}$, entre visitantes $\left(23,4-25,5^{\circ} \mathrm{C}\right)$ e para equipe de saúde $\left(24,6-26,8^{\circ} \mathrm{C}\right)$. Ademais, foi observado que a temperatura neutra variou mais entre pacientes, em detrimento, ao grupo dos visitantes e equipe de saúde, além disso, há uma menor quantidade de pesquisas centrada nos visitantes.

Quando se compara o modelo baseado pelo TSV e o PMV por grupo (paciente, visitante e equipe de saúde), nota-se que, apesar da variação de temperatura neutra para equipe de saúde e visitante ser maior pelo PMV, quando o foco são os pacientes, percebe-se que a variação foi maior pelo TSV. Isso pode ser explicado, pela maior variação de percepção de temperatura apresentada entre os pacientes, uma vez que há maiores mudanças na taxa metabólica e de isolamento térmico por roupas, cama, entre outros. Assim, o TSV foi capaz de demonstrar com maior precisão essas alterações, o que corrobora com pesquisas anteriores (ALOTAIBI et al., 2020).

Tabela 2: Dados dos trabalhos incluídos na revisão sistemática

\begin{tabular}{|c|c|c|c|c|c|c|c|c|c|c|c|}
\hline \multirow{3}{*}{ Pesquisadores } & \multirow{3}{*}{ Ano } & \multirow{3}{*}{ Local } & \multirow{3}{*}{$\begin{array}{l}\text { Qnt. } \\
\text { Part }\end{array}$} & \multicolumn{6}{|c|}{ Temperatura Neutra $\left({ }^{\circ} \mathrm{C}\right)$} & \multirow{3}{*}{$\begin{array}{l}\text { Variação } \\
\text { temperatura } \\
\text { neutra }\end{array}$} & \multirow{3}{*}{$\begin{array}{l}\text { Satisfação } \\
80 \%\end{array}$} \\
\hline & & & & \multicolumn{3}{|c|}{ PMV Based } & \multicolumn{3}{|c|}{ TSV Based } & & \\
\hline & & & & Paciente & Visitante & Staff & Paciente & Visitante & Staff & & \\
\hline $\begin{array}{l}\text { KHODAKARA } \\
\text { MI et al }\end{array}$ & 2008 & Irã & - & $\begin{array}{l}22,5-28 \\
27-31,5\end{array}$ & - & $19-26$ & - & - & - & - & $24-26^{\circ} \mathrm{C}$ \\
\hline $\begin{array}{l}\text { NEMATCHUO } \\
\text { A }\end{array}$ & 2017 & $\begin{array}{l}\text { Madagas } \\
\text { car }\end{array}$ & 298 & 25,46 & - & - & 26,41 & - & - & 0,95 & $23,2-26,8$ \\
\hline $\begin{array}{l}\text { SATTAYAKO } \\
\text { RN et al }\end{array}$ & 2017 & $\begin{array}{l}\text { Tailândi } \\
\text { a }\end{array}$ & 928 & 25,3 & 25,4 & 22,4 & 25,2 & 25,1 & 25,5 & 3,1 & $\begin{array}{l}21,8-27,9 \\
22-27,1 \\
24,1-25,6\end{array}$ \\
\hline YAU e CHEW & 2009 & Malásia & 114 & - & - & 26,4 & - & - & 26,4 & - & $25,3-28,2$ \\
\hline HWANG et al. & 2007 & Taiwan & 491 & 25,6 & - & - & 23,4 & - & - & 2,2 & $21,8-26,2$ \\
\hline $\begin{array}{l}\text { AZIZPOUR et } \\
\text { al }\end{array}$ & 2013 & Malásia & 188 & - & 21,3 & & - & 23,4 & & 2,1 & $21,2-25,5$ \\
\hline $\begin{array}{l}\text { AZIZPOUR et } \\
\text { al }\end{array}$ & 2012 & Malásia & 110 & - & - & 25,0 & - & - & 26,8 & 1,8 & - \\
\hline WANG et al & 2012 & Taiwan & 403 & - & - & $\begin{array}{l}23,6- \\
27,9\end{array}$ & - & - & $\begin{array}{l}22,9- \\
26,3 \\
(\mathbf{2 4 , 6 )}\end{array}$ & 1 & $22,9-26,3$ \\
\hline KHALID et al & 2019 & Malásia & 389 & - & - & - & 25,3 & 25,5 & - & - & $\begin{array}{l}\operatorname{Max} 27,7 \\
\operatorname{Max} 26,9\end{array}$ \\
\hline ALOTAIBI et al & 2020 & $\begin{array}{l}\text { Arábia } \\
\text { Saudita }\end{array}$ & 120 & 25,6 & - & - & 22,7 & - & - & $-2,9$ & $16,2-28,8$ \\
\hline
\end{tabular}

É importante identificar a composição dos pacientes, profissionais e visitantes a quem se direciona, sempre que possível, uma vez que a variação de gênero, idade, tipo de doença crônica (queimados, DRC, politraumatizado) pode alterar as necessidades e preferencias dos pacientes. Nos casos de desequilíbrio orgânico/homeostático, demonstrou-se a preferência de temperaturas mais quentes pelos pacientes, em detrimento, àquelas esperadas pelos funcionários e visitantes.

Verificou-se no grupo dos profissionais de saúde a maior discordância possível entre a temperatura neutra esperada pelo PMV e o TSV, uma vez que os parâmetros da ASHRAE-55 esperavam, a partir da maior atividade e isolamento térmico das roupas, temperaturas bem mais baixas, não condizentes com as demonstradas na avaliação subjetiva (TSV) (AZIZPOUR et al., 2012; SATTAYAKORN et al., 2017). Caso seja necessário, sugere-se o controle de temperatura diferenciado na sala de prescrição, posto de enfermagem e copas. A maioria das pesquisas relatou a preferência da população por uma temperatura levemente mais fria, cerca de $-1^{\circ} \mathrm{C}$, àquela apresentada pela ASHRAE.

Uma grande parcela da energia gasta em hospitais tem a finalidade de controlar a temperatura 
interna dos ambientes, sobretudo, quando utilizado aparelhos de refrigeração. Os estudos defendem que seja preferível o uso de ventiladores elétricos no topo das salas a uso de aparelhos refrigeradores, uma vez que gasta menos energia e leva a um conforto térmico maior aos usuários, que reclamam das temperaturas excessivamente baixas. Ademais, reforça-se o uso do sistema HVAC como mecanismo de redução de custos.

Assim, pode-se calcular a temperatura máxima confortável para, pelo menos, $80 \%$ das pessoas e utilizar esse valor como alvo. Dessa forma, podemos almejar em países tropicais e subtropicais, temperaturas mais altas sem afetar o conforto e garantindo, assim, menores gastos energéticos.

Pode ser usado um controle mais rígido nos quartos de pacientes mais frágeis devido a suas necessidades mais específicas e, assim, diminuindo os gastos energéticos nos demais ambientes. Bem como naqueles que necessitam de controle de higiene, devido ao uso de equipamentos invasivos no paciente ou a falta de capacidade do hospital em trocar as roupas de cama e do paciente. Ademais, pacientes com altos níveis de ansiedade e estresse sofreram maiores variações na sua percepção térmica ideal (SATTAYAKORN et al., 2017).

Pacientes com capacidade de alterar seus níveis de isolamento térmico através das roupas, demonstraram maior satisfação térmica, adaptando-se ao ambiente apresentado. Apesar disso, evidenciase que pacientes no geral possuem menor capacidade de adaptação a temperatura do ambiente.

Sabe-se que hospitais estão densamente ocupados por pacientes de idade mais altas, haja vista que os idosos adoecem com maior facilidade. Além disso, esse grupo possui capacidade termorregulação pelo hipotálamo diminuída e, frequentemente, limitação para atividades diárias como tomar banho, trocar de roupa e comer. Assim, esses pacientes estão mais vulneráveis e apresentam necessidade de controle térmico mais rígido.

Deve-se isolar portas e janelas, instalar cortinas nas janelas para impedir o aumento da temperatura pela radiação solar. Deste modo, seria possível, também, alterações de temperatura do ambiente pelos próprios ocupantes do espaço, através do controle das variáveis térmicas como o fluxo de ar e a radiação solar. Há uma necessidade de variação da temperatura neutra em pacientes com doença crônica de 0,3 a $0,6^{\circ} \mathrm{C}$ (YAU et al., 2009).

Houve uma associação positiva entre pessoas do sexo masculino e a preferência por temperaturas mais elevadas, variando nessas pesquisas entre 0,6 a 1,3 ${ }^{\circ} \mathrm{C}$ (NEMATCHOUA et al., 2007; ALOTAIBI et al., 2020). Compreende-se que há variações significativas de temperatura neutra, de acordo com a estação do ano, sendo indicada a avaliação de mais estudos para saber como se comportam (HWANG et al., 2007).

\section{CONCLUSÕES}

Dessa forma, observa-se que os padrões de temperatura da ASHRAE e ISO 7730 não condizem com as expectativas dos habitantes dos trópicos. Assim, faz-se necessário mais estudos para que se possa alcançar melhor adequação, sendo preferível que seja realizado em cada local.

Apesar dos desafios apresentados aos arquitetos, designers e engenheiros para adaptar o ambiente aos padrões de temperatura esperados, é importante projetá-lo racionalizando as características e 
necessidades dos pacientes, profissionais de saúde e visitantes que irão frequentar, bem como dos gastos energéticos e suas roupas, haja vista as variações de temperatura neutra que irão necessitar.

Deve-se considerar a possibilidade de avaliar a temperatura máxima com $80 \%$ dos pacientes confortáveis, quando não for obrigatório controles rígidos. Uma vez que permitiria o menor gasto energético, além de utilizar mais de técnicas de ventilação natural, cortinas, ventilador no teto e HVAC, ao invés de apenas aparelho refrigerador.

Pode ser projetado os ambientes direcionados aos funcionários do hospital com conformidades térmicas distintas, visando respeitar as particularidades desse grupo. Bem como, os pacientes que possuírem necessidades metabólicas rígidas ou vulnerabilidade social, dado as suas limitações físicas, mentais e/ou homeostáticas.

\section{REFERÊNCIAS}

ABBASI, A. M.; MOTAMEDZADE, M.; ALIBADI, M.; GOLMOHAMMADI, R.; TAPAK, L.. Study of the physiological and mental health effects caused by exposure to low frequency noise in a simulated control room. Building Acoustics, v.25, n.3, p.233-248, 2018. DOI: http://doi.org/10.1177/1351010X18779518

ALOTAIBI, B.; LO, S.; SOUTHWOOD, E.; COLEY, D.. Evaluating the suitability of standard thermal comfort approaches for hospital patients in air-conditioned environments in hot climates. Building and Environment, v.169, n.106561, 2020. DOI: http://doi.org/10.1016/j.buildenv.2019.106561

ASHRAE. American Society of Heating, Refrigerating and AirConditioning Engineers. ANSI/ASHRAE Standard 55-2013: Thermal Environmental Conditions for Human Occupancy. Atlanta: ASHRAE, 2013.

AZIZPOUR, F.; MOGHIMI, S.; LIM, C.; MAT, S.; SALLEH, E.; SOPIAN, K.. A thermal comfort investigation of a facility department of a hospital in hot-humid climate: Correlation between objective and subjective measurements. Indoor Built Environ., v.22, p.836-845, 2012. DOI: http://doi.org/10.1177/1420326X12460067

AZIZPOUR, F.; MOGHIMI, S.; SALLEH, E.; SOHIF, E.; SOHIF, M.; LIM, C.; SOPIAN, K.. Thermal comfort assessment of largescale hospitals in tropical climates: A case study of University Kebangsaan Malaysia Medical Centre (UKMMC). Energy and Buildings., v.64, p.317-322, 2013. DOI: http://doi.org/10.1016/j.enbuild.2013.05.033

CASTALDO, V. L.; PIGLIAUTILE, I.; ROSSO, F.; GIORGIO, F.; PISELLO, A.. How subjective and non-physical parameters affect occupants' environmental comfort perception. Energy and Buildings, v.178, p.107-129, 2018. DOI: https://doi.org/10.1016/i.enbuild.2018.08.020

CORBELLA, O.; YANNAS, S.. Em busca de uma arquitetura sustentável para os trópicos. Rio de Janeiro: Revan, 2003.

DEHGHAN, H.; AZMOON, H.; SOURI, S.; AKBARI, J.. The effects of state anxiety and thermal comfort on sleep quality and eye fatigue in shift work nurses. J. Educ. Health. Promot., v.3, p.72, 2014. DOI: http://doi.org/10.4103/ $\underline{2277-9531.134870}$
DOVJAK, M.; SHUKUYA, M.; KRAINER, A.. User-Centred Healing-Oriented Conditions in the Design of Hospital Environments. Int. J. Environ. Res. Public. Health, v.15, n.10, p.2140, 2018. DOI: http://doi.org/10.3390/ijerph15102140

FERNANDES, A. M. C. P.. Clima, Homem e Arquitetura. Goiânia: Trilhas Urbanas, 2006.

FRANSSON, N.; VÄSTFJÄLL, D.; SKOOG, J.. In Search of the Comfortable Indoor Environment: a comparison of the utility of objective and subjective indicators of indoor comfort. Building and Environment, v.42, n.5, p.1886-1890, 2007. DOI: https://doi.org/10.1016/j.buildenv.2006.02.021

FRONTCAZK, M., ANDERSEN, R., WARGOCKI, P..

Questionnaire survey on factors influencing comfort with indoor environmental quality in Danish housing. Building and Environment, v.12, p.56-64, 2012. DOI: https://doi.org/10.1016/j.buildenv.2011.10.012

GONZALEZ, M. S. R.; FERNANDEZ, C. A.; CAMESELLE, J. M. S. Empirical validation of a model of user satisfaction with buildings and their environments as workplaces. Journal of Environmental Psychology, v.17, p.69-74, 1997. DOI: https://doi.org/10.1006/jevp.1996.0040

HAGHIGHAT, F.; DONNINI, G.. Impact of psychosocial factors on perception of the indoor air environment studies in 12 office buildings. Building and Environment, v.34, p.479-503, 1999. DOI: https://doi.org/10.1016/S0360-1323(98)00034-1

HASHIGUCHI, N.; HIRAKAWA, M.; TOCHIHARA, Y.; KAJI, Y.; KARAKI, C.. Thermal environment and subjective responses of patients and staff in a hospital during winter. J. Physiol. Anthropol Appl. Human Sci., v.24, n.1, p.111-115, 2005. DOI: http://doi.org/10.2114/ipa.24.111

HAVENITH, G.. Temperature regulation and technology. Gerontechnology, v.1, p.41-49, 2001. DOI: http://doi.org/10.4017/gt.2001.01.01.004.00

HEAKYUNG, C. Y.. Differences in performance with different background sound and ambient noise in three open office plans J. Acoust. Soc. Am., v.108, p.2632, 2000. DOI: http://doi.org/10.1121/1.4743797 
HOOF, J.. Forty years of Fangers model of thermal comfort: comfort for all?. Indoor Air, v.18, p.182-201, 2008. DOI: https://doi.org/10.1111/j.1600-0668.2007.00516.x

HORR, Y.; ARIF, M.; KATAFYGIOTOU, M.; MAZROEI, A.; KAUSHIK, A.; ELSARRAG, E.. Impact of indoor environmental quality on occupant well-being and comfort: A review of the literature. International Journal of Sustainable Built Environment, v.5, n.1, p.1-11, 2016. DOI: http://doi.org/10.1016/j.ijsbe.2016.03.006

HUMPHREYS, M.; NICOL, F.. Understanding the adaptive approach to thermal comfort. ASHRAE Transactions, v.98, n.1, p.991-1004, 1998.

HUMPHREYS, M.. Quantifying occupant comfort: are combined indices of the indoor environment practicable? Build Res. Inf., v.33, p.317-325, 2005. DOI: https://doi.org/10.1080/09613210500161950

HWANG, R.; LIN, T.; CHENG, M.; CHIEN, J.. Patient thermal comfort requirement for hospital environments in Taiwan. Building and Environment, v.42, p.2980-2987, 2007. DOI: https://doi.org/10.1016/j.buildenv.2006.07.035

KARJALAINEN, S.. Gender differences in thermal comfort and use of thermostats in everyday thermal environment. Build Environ., v.42, p.1594-1603, 2007. DOI: https://doi.org/10.1016/i.buildenv.2006.01.009

KHALID, W.; ZAKI, A.; RIJAL, H.; YAKUB, F.. Investigation of comfort temperature and thermal adaptation for patients and visitors in Malaysian hospitals. Energy and Buildings. v.183, p.484-499, 2019. DOI:

http://doi.org/10.1016/j.enbuild.2018.11.019

KOSONEN, R.; TAN, F.. PMV-derived productivity model as a tool to assess productivity loss. In: THAM, K., SEKHAR, S.. Proceedings of Healthy Buildings. Singapore, 2003. p.749754.

ISO. International Organization for Standardization. ISO 7730: Ergonomics of the Thermal Environment: Analytical Determination and Interpretation of Thermal Comfort Using Calculation of the PMV and PPD Indices and Local Thermal Comfort Criteria. Vernier: ISO, 2005.

KHODAKARAMI, J.; KNIGHT, I.. Required and Current Thermal Conditions for Occupants in Iranian Hospitals. HVAC\&R Research, v.14, n.2, p.175-193, 2008. DOI: http://doi.org/10.1080/10789669.2008.10391002

LAN, L.; THUSAR, W.; OTTO, K.; WOOD, K.. Thermal comfort improvement of naturally ventilated patient wards in Singapore. Energy and buildings, v.154, p.499-512, 2017. DOI: https://doi.org/10.1016/i.enbuild.2017.07.080

MACNAUGHTON, P.; SATISH, U.; LAURENT, J.; FLANIGAN, S.; VALLARINO, B.; SPENGLER, J.; ALLEN, J.. The impact of working in a green certified building on cognitive function and health, Building and Environment, v.114, p.178-186, 2017. DOI: https://doi.org/10.1016/j.buildenv.2016.11.041

MENDELL, M. J.; FISK, W. J.; KREISS, K.; LEVIN, D.; ALEXANDER, W. S.; CAIN, J. R.; HINES, C. J.; JENSE, P. A.; MILTON, D. K.; REXROAT, L. P.; WALLINGFORD, K. M..
Improving the health of workers in indoor environments: priority research needs for a national occupational research agenda Am. J. Public Health, v.92, n.9, p.1430-1440, 2002. DOI: http://doi.org/10.2105/ajph.92.9.1430

MUSSI, F. C.. Conforto: revisão de literatura. Rev. Esc. Enf. USP, v.30, n.2, p.254-66, 1996. DOI:

https://doi.org/10.1590/S0080-62341996000200006

NEMATCHOUA, K.; RICCIARDI, M.; REITER, P.; ASADI, S.; DEMERS, C.. Thermal comfort and comparison of some parameters coming from hospitals and shopping centers under natural ventilation: The case of Madagascar Island. Journal of Building Engineering, v.13, p.196-206, 2017. DOI: http://doi.org/10.1016/j.jobe.2017.07.014

OCHOA, J.; ARAUJO, D.; SATTLER, M.. Análise do conforto ambiental em salas de aula: comparação entre dados técnicos e a percepção do usuário. Ambiente Construído, v.12, n.1, p.91-114, 2012. DOI: https://doi.org/10.1590/S1678-86212012000100007

OLESEN, B.; NIELSEN, R.. A comparison of the termal insulation measured on a termal manikin ando $\mathrm{n}$ human subjects. In: BERGLUND, B.; LINDVALL, T.. Proceedings of Indoor Air 84. 5 ed. 1984. p.315-320.

POURSHAGHAGY, A.; OMIDVARI, M.. Examination of thermal comfort in a hospital using PMV-PPD model. Applied Ergonomics, v.43, n.6, p.1089-1095, 2012. DOI: http://doi.org/10.1016/j.apergo.2012.03.010

ROELOFSEN, P.. The impact of office environments on employee performance: The design of the workplace as a strategy for productivity enhacement. Journal Facilities Management, v.1, n.3, p.247-263, 2002. DOI: http://doi.org/10.1108/14725960310807944

SANTOS, M.. A Natureza do Espaço: Técnica e Tempo, Razão e Emoção - 1926. 4 ed. São Paulo: Universidade de São Paulo, 2006. DOI: https://doi.org/10.22409/GEOgraphia2000.v2i3.a13382

SANTOS, M.. Pensando o espaço do homem. 5 ed. São Paulo: EDUSP, 2009.

\section{SANTOS, M.. Metamorfoses do Espaço Habitado:}

fundamentos teóricos e metodológicos da geografia. 6 ed. São Paulo: Universidade de São Paulo, 2014.

SATTAYAKORN, S. ICHIONOSE, M.; SASAKI, R.. Clarifying thermal comfort of healthcare occupants in tropical region: A case of indoor environment in Thai hospitals. Energy and Buildings, v.149, p.45-57, 2017. DOI: https://doi.org/10.1016/j.enbuild.2017.05.025

SATTAYAKORN, S.; ICHINOSE, M.; SASAKI, R.. Comfort in patient room of healthcare facilities in tropical region: $A$ different requirement between patient and their comparison. In: PLEA INTERNATIONAL CONFERENCE ON DESIGN TO THRIVE, 33. Anais. 2017. p.1273-1280.

SKOOG, J.; FRANSSON, N.; JAGEMAR, L.. Thermal environment in Swedish hospitals: Summer and winter measurements. Energy and Buildings, v.37, p.872-877, 2005. DOI: https://doi.org/10.1016/j.enbuild.2004.11.003 
SOUZA, E. C. V.. Legislação sustentável: diretrizes para incorporação de conceitos de sustentabilidade no código de edificações de vitória/ES. Revista do Programa de PósGraduação em Arquitetura e Urbanismo da FAAUSP, v.22, n.38, p.124-139, 2015. DOI:

https://doi.org/10.11606/issn.2317-2762.v22i38p124-139

VIOLA, A. U.; JAMES, L. J.; FAU-SCHLANGEN, L. M.; SCHALANGEN, J.; DIJK, L.. Blue-enriched white light in the workplace improves self-reported alertness, performance and sleep quality. Scand J. Work Environ Health, v.34, n.4, p.297-306, 2008. DOI: http://doi.org/10.5271/sjweh.1268

WANG, F.; LEE, M.; CHENG, T.; LAW, Y.. Field evaluation of thermal comfort and indoor environment quality for a hospital in a hot and humid climate, HVAC\&R Research, v.18, n.4, p.671-680, 2012. DOI:

http://doi.org/10.1080/10789669.2012.644102

WHO. World Health Organization. Environmental Health in
Rural and Urban Development and Housing Unit, Indoor environment: health aspects of air quality, thermal environment, light and noise. Geneva: WHO, 1990.

YANG, W., MOON, H.. Combined effects of acoustic, termal, and illumination conditions on the comfort of discrete senses and overall indoor environment. Building and Environment, v.148, p.623-633, 2019. DOI: https://doi.org/10.1016/j.buildenv.2018.11.040

YANG, W.; ZHANG, G.. Thermal comfort in naturally ventilated and air-conditioned buildings in humid subtropical climate zone in China. International Journal of Biometeorology, v.52, n.5, p.385-98, 2008. DOI: https://doi.org/10.1007/s00484-007-0133-4

YAU, Y.; CHEW, B.. Thermal comfort study of hospital workers in Malaysia. Indoor Air, v.19, p.500-510, 2009. DOI: http://doi.org/10.1111/j.1600-0668.2009.00617.x

A CBPC - Companhia Brasileira de Produção Científica (CNPJ: 11.221.422/0001-03) detém os direitos materiais desta publicação. Os direitos referem-se à publicação do trabalho em qualquer parte do mundo, incluindo os direitos às renovações, expansões e disseminações da contribuição, bem como outros direitos subsidiários. Todos os trabalhos publicados eletronicamente poderão posteriormente ser publicados em coletâneas impressas sob coordenação da Sustenere Publishing, da Companhia Brasileira de Produção Científica e seus parceiros autorizados. Os (as) autores (as) preservam os direitos autorais, mas não têm permissão para a publicação da contribuição em outro meio, impresso ou digital, em português ou em tradução. 